\title{
Les langues turcques et quelques questions de l'histoire des Tchouvaches
}

\author{
Dr. Anton K. SALMIN \\ Musée d'anthropologie et d'ethnographie nommé d'après Peter le grand (Kunstkamera) \\ Académie des sciences de Russie \\ L'histoire était extrêmement impitoyable \\ pour les Chuvash et leur langue. Pendant \\ plusieurs siècles, ils resté dans l'oubli et \\ l'obscurité complete comme dans la position \\ d'un peuple sans attaches ni traditions
}

( Egorov 1953: 66).

\begin{abstract}
The language also contains history. For analysis, it must be compared with related languages and languages of neighbouring tribes and peoples. The language can split into several descendant languages over time. This is due to violation of the unity of the society in which it operates. When differences begin to complicate mutual understanding between the members of society, then different but related languages appear. Change is an obligatory feature of the language, if only because a static system could not provide communication of the society in the changing world. Linguists (A.V. Dybo et al.) established the washing rate of 5 words from the Swadesh list over 1,000 years on a number of language groups. With such rate for 20,000 years, Swadesh list should wash out completely. It turns out there are no static languages that have existed for 20,000 years. The Turkic language is a superstrate language, and not a substrate in any way for the modern Chuvash language, that is, this process occurred (and continues to take place) not in Siberia, but in the Volga region.
\end{abstract}

Keywords: Turkic languages, the Chuvash, history, descendant languages, V.V. Radlov

\section{Introduction}

Composants ethniques nes sont pas les signes permanentes. Le changement des composantes culturelles les aident à se transformer, à surmonter les crises et à promouvoir la renaissance. Au sens le plus large les peuples se renaissent. Les tribus turco-mongoles, soit se réunissant, se désintégrant maintenant en hordes, soit entraînant des tribus conquises dans leurs unités militaires, ont pu conquérir les steppes. Ainsi, certaines nations ont disparu et d'autres sont apparues.

\section{La partie principale}

Le lieu d'existence de la proto-langue turcque était le vaste territoire situé entre Ordos et le sud Sayano-Altai. Le périod d'un tel état de langage est considéré comme la fin du millénaire avant notre ère - les premiers siècles après JC. En ce moment-là la langue ancienne turque s'est séparée des familles mongoles et tungus-mandchoues (Dybo 2007: 199-200). Même pendant l'existence de l'ancienne langue turque (ancien turc), estime Andras Rona-Tash, les locuteurs de l'ancienne langue tchouvache se sont éloignés des autres Turcs. Les linguistes divisent les langues turcques en deux groupes principaux: les langues Ogur et Oguz. Tous ont un marqueur de pluralité -лар sauf le Tchouvache. Ce généralisation a apparu plus tard, parce que la langue turcque ancienne avait les autres formes plurielles. Cela se voit à partir d'exemples tels que og̉ul "fils" > oglan "fils", är "homme"> ärän "hommes" et également be (n) "je" > biz "nous" (Róna-Tas 2007). En tchouvache, le suffixe pluriel est -cam/-cem et en bashkir, - -map/-mәр, -дар/-дәр, -зар/-зәр.

Dans les annales dynastiques chinoises et sur les inscriptions turcque, un certain nombre de titres et de posts sont mentionnés, indiquant leurs emprunts à Juan Juans de sources chinoise, iranienne, tokharienne et indienne. Par exemple, yabğu, šad, tegin, ilteber, tudun, tutuq, khagan, khan, tegin, chor, tarkhan et autres. En général, grande majorité des premiers titres et noms turques (Bek, Bumyn, Istemi, Mukan, Taspar, Nevar) sont d'origine non turque (Golden 2006: 17-38; Zhivkov 2015: 23-24).

On pense que la langue Ogure / Bulgare est une forme antérieure de turcque archaïque et seulement par la suite une série de changements phonétiques ont eu lieu (c'est-à-dire $z>r, * l \check{s} / * l \check{c}>l$ ). Probablement c'est passé dans le I III siècles. Les rudiments de cette proto-langue sont préservés chez Tchouvashes jusqu'à présent (Golden 2011: 30). Cependant, pas toutes les inscriptions d'Orkhon appartenaient aux tribus turques, particulièrement aux Tatars malgré que devant leur nom on met les chiffres turcques comme отуз "trente" et токуз "neuf". 
Wilhelm Thomsen considérait que ces Tatars étaient des Mongols (Thomsen 1924: 174). V.V. Barthold était entièrement d'accord avec lui (Barthold 1968: 580). À cette époque, les steppes de l'Europe de l'Est étaient habitées par des nomades de langue iranienne tels que les Scythes, les Sarmates et les Alains. À partir de VI siècle les éléments turcophones ont commencé à prendre de l'essor. À la basé de l'étude analytique de la manifestation du rhotacism et du lambdaïsm dans les langues des groupes Ogur et Tchouvache Andrush Rona-Tash indique que les personnes / groupes parlant le tchouvache (Chuvash speaking people/groups) venaient du Sud de la Sibérie. Par

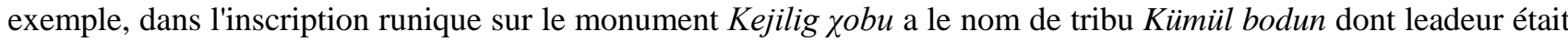
Kümül üge. Ce leadeur est devenu üge à l'âge de 30 ans (otuz yašimga) et est décédé à l'âge de 70 ans (yetmiš yašimga). Sans aucun doute le texte est écrit dans le vieux turcque commun, mais le lambdaïsme prend forme dans le mot kümül «argent» (moyen Tchouvache кёмёл et Turcque commun Kümüšs). Il est remarquable que ces "gens d'argent" parlaient langue du type tchouvache et ce nom en est préservé aux VIII et IX siècles. Le «gens d'argent» (kümül bodun) sont également mentionnés dans l'inscription Kizil Čira et dans les sources chinoises. Telles preuves linguistiques montrent que les anciens groupes parlant la langue tchouvache en Sibérie du Sud étaient divisés, la plupart d'entre eux s'étant rendus en Europe, mais certains sont restés en Asie et ont ensuite été assimilés par les Turcs qui y vivaient (Róna-Tas 2007). Dans l'inscription Yenisei Кюмюль Оге est un nom masculin (militaire), son propriétaire appelle кюмюльей "mon peuple". Apparemment, ils constituaient de nombreux clans, car Кюмюль Оге appelle son peuple également «cent kumyuly» (Aydın 2011: 7-8).

Les changements principaux de la langue et de la culture de l'Europe orientale se sont déroulés au Moyen Âge. Aux XII-XVe siècles, la steppe était remplie par les tribus turcqophones. L'élite dirigeante mongole n'est pas adaptée parmi les citoyens et a rapidement été turcquisée. «Le nom occidental des Mongols - Tatars - au XIVe siècle désignait des personnes qui parlaient kipchak» (Zimonyi 2007). La source la plus importante dans la langue Kypchak est "Codex Cumanicus" composé au XIVe siècle sur le terrain. La langue des principaux successeurs du kuman (kipchak occidental) les Tatars de la Volga n'a pas changé en termes phonétique à ce jour.

Un certain nombre de chercheurs cherchent activement des arguments pour donner aux ancêtres des Tchouvaches un ancien substrat turcque. Il y avait toujours suffisamment de raisons car le terme tatars était un concept collectif. Même à la fin du XVIIe siècle Nikolaas Vitsen et d'autres sources appellent les les Tchouvaches comme les Tatares (ainsi que tous les peuples finno-ougriens)

Non loin de Kazan un petit peuple tartare vive... Ce peuple s'appelle Chuvash (Vitsen 2010: 906).

Cependant, les tartres dans ce contexte ne sont plus que étrangers.

$\mathrm{Ne}$ trouvant pas de preuves suffisantes de la présence du substrat turcque aux ancêtres des Chuvash, les chercheurs font appel aux connexions de Saviro-Hunnic. Donc il n'y a pas de premiers faits marquant le turcquisme.

Les opinions erronées sur la datation antérieure de ce phénomène sont encore rencontrées dans la littérature le plus souvent basées sur des hypothèses souhaitables, et non sur des faits solides scientifique. Malheureusement, tous ne sont pas encore conscients et convaincus que les Huns, comme les tribus du Caucase du Nord, ne parlaient pas la langue turcque, mais c'était exactement comme ça (Kyzlasov 1997: 27-28).

On sait que le Khaganate turque a été créé par les dirigeants Bumyn et Istemi du clan Ashina, au milieu VI siècle. En 567, Istemi a envoié Mikhail Sogdiy à Byzance pour établir les relations diplomatiques. L'itinéraire de l'ambassade traversait l'Asie centrale et le Caucase. À son retour, Michael était accompagné par l'ambassadeur de Byzantin, Zemarhos (Zimarkh, Zemarh) (Rtveladze 2015: 357-363). Évidemment, la diplomatie ne pouvait pas se passer d'écriture, on suppose que alphabet runique a été créé à cette fin. À cet égard, on suppose que les Turcques ont utilisé l'alphabet iranien et y ont ajouté des lettres grecques pour désigner les voyelles de la première rangée. En 576, les troupes turques ont défait les Byzantins sur les rives du Bosphore et se sont installées en Crimée et dans le Caucase du Nord.

Le "dictionnaire ancien turcque", le plus autorisé, contient le vocabulaire des VII-XIII siècles. Prenons le premier mot de ce dictionnaire, le mot le plus cher - aba au sens de "mère", "père", "progéniteur" (Nadelyayev et al. 1969: 1). $A b a$ au sens de "mère" est tiré du dictionnaire de Mahmud Kashgar. Aba comme «père» était inclus dans la langue turcque à partir des langues tibétaines et a également été enregistré par Mahmud Kashgar. $A b a$ au sens de "progéniteur", "ancêtre" venait de la langue arabe. Le temps d'enregistrement de tous les aspects du mot aba est XI siècle. Ce mot existe dans le dialecte supérieur de la langue tchouvache sous la forme anaŭ (en abaj) et signifie «ma mère», «ma belle-mère», «la sœur aînée de mon mari», «grand-mère paternelle». Il est apparu dans la langue Tchouvache au mieux du XIIIe siècle. Dans le même sens il existe des mots dans la langue атmе «père», анне «mère», хуняма «belle-mère», асанне «grand-mère», littéralement la «mère aînée» et ainsi ana n'existe maintenant que dans le nord-ouest de la République de Tchouvache. Le nom dérivé est abučqa ("vieil homme"). Tchuvash упӑшка qui signifie «mari», «vieil homme» revient au même mot $a b a$ «père» Il y a ce mot dans le dialecte GornoMari sous la forme d'une овылка «veuve» qui était emprunte de la langue tchouvache. On peut continuer à énumérer les exemples. Par exemple, le mot arabe adam "homme" figure également dans la liste du vocabulaire du turcque ancien.

106 
Ce mot persane était emprunté par plusieurs langues Caucasienne (Chamalal, Lak, Lezgian, Tabasaran, Tsakhur,Rutul, Budukh, Udmurt, Khinalug, Aghul, Tchetchen, Andi, Karata, Bagwalal, Botlikh, Godoberi, Tsez, Bejta, Hunzib, Hinukh, Dargwa, Digor) et ont épousé la forme de hadam, adam, ädäm, ädämi, adaw, adamina, itim, adami, admi, idemi, edemi, idimi (Takazov 2015: 131). Le mot etem est venu dans la langue tchouvache du persane (Dybo 2015: 419) $\rightarrow$ Edem Gorno-Mari et il y a une foison d'exemples. Par conséquent, il est très inconsidérément de dire que l'ancien vocabulaire turcque est le fonds lexical du vieux tchouvache. Et le «dictionnaire ancien turcque» lui-même est un dictionnaire consolidé de la langue des âges médiévaux, pas des anciens dialectes turcques. En même temps, il contient une série de mots dont l'origine, la chronologie et le lieu sont presque impossible de déterminer. Par exemple suffixe er au sense «homme», «mari», «conjoint». Ce mot est fixé dans l'inscription Orkhon-Yenisei en 732 (Nadelyayev et al. 1969: 175). Le mot se retrouve dans toutes les langues turcque et mongole, ainsi que dans les langues sumérienne et tchuvache, sous les formes $a p$, $\ddot{p} p, \ni p, u p$, эре. Très probablement, ce mot est d'origine aryen. Même le mot тимёр «fer» perçu maintenant comme à l'origine turcque remonte au chinois tiet-mür et le nom de Temujin "forgeron" a la même origine. Et Gengis Khan était illettré et ne connaissait pas une seule langue à l'exception de sa langue mongole.

Il y a plein de mots d'origine persane sont inclus dans la langue tchouvache par l'intermédiaire du turcque. Le mot tchouvache naxya "jardin" remonte au bag persan "jardin", "vignoble". Dans le dialecte du nord de la langue tchouvache une forme antérieure (persane) sous la forme de пахулми «pomme du jardin» a été conservée contrairement à с̧ӗр улми «pomme de terre». Le mot persan "jardin" ont été enregistrés dans les langues

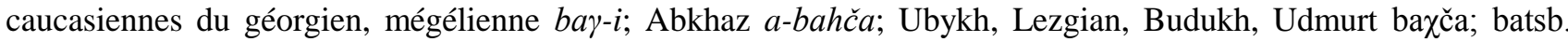
baqč. On pense que dans les langues modernes du Caucase, le mot a été répandu à travers les Turcques (Klimov, Khalilov 2003: 157). Quant au nom du village de Bakhtiyarovo il est d'origine persane (Bakhtiyar est un prénom masculin), mais il est apparemment entré dans la langue tchouvache par des moyens turcques car ce nom est populaire parmi les peuples turcophones. Le mot tchouvache тёмен au sens de «paix», «ensemble» est un emprunt moyen iranien. Source - Persain tümān "dix mille". Le chiffre est clairement associé au nom de l'unité militaire dans les langues turques.

Gustav Ramstedt dans ses travaux sur la place de la langue tchouvache dans la famille des langues ouralo-altaites indique que le lambdaïsm et le rotacisme dans les langues toungouse, mongole et tchouvache sont phénomène préturcque (Ramstedt 1922: 32). Il a classé les langues turque et tchouvache aux branches linguistiques occidentales du Hunn et du Sud-Hunn, ce qui doit être clarifié à la lueur de récents données dans l'étude de l'histoire des relations Hun-Savir. N.N. Poppe et V.V. Barthold ont affirmé que la langue tchouvache avait été formée à une époque où les caractéristiques des langues du groupe turcique n'avaient pas encore été établies. V.V. Barthold également pensait que l'opposition turque-tchouvache sous la forme d'une transition phonétique $p>3$ et $\pi>m$ se produisait vers le début de l'époque (Barthold 1968: 198, 579). Mais les premiers contacts des locuteurs des langues chuvashe et turcque ne devraient pas être attribués avant la seconde moitié du VIe siècle, lorsque les détachements d'avant-garde des tribus turciques ont rencontré les Savirs en Europe. Il y a les autres faits fondamentales attestent que la langue tchouvache est un phénomène pré-turcque. Par exemple, les inscriptions tombales en Bulgarie Volgienne reflètent bien aux dialectes $p$ et $l$ de la langue tchouvache, par opposition aux dialectes 3 et $u$ des langues turques. Ici nous avons les mots mухур "neuf", саккар "huit", вутур "trente", хир "fille", арня кун "vendredi", биалем "cinquième". Ces mots dans d'autres langues turcophones ressemblent aux тугыз, секиз, кыз, атна кон, бишенче. Cela devrait également inclure des exemples du type çăltăr - julduz "étoile", "planète". En général, le son turc $z$ correspond non seulement à la Tchouvache, mais également au $r$ Mongol. En réfléchissant à une telle situation phonétique Andras Rona-Tash dit:

Qu'est-ce qui a empêché l'existence de l'opposition $r: z, s ̌$ : l? ". Le linguiste hongrois suggère l'influence d'une telle langue dans laquelle l'opposition $r: z, \breve{s}: l$ n'existait pas. Il considère le prongol comme cette langue substrat (RónaTas 1970: 209: 228).

Si cette hypothèse est correcte, il s'agit d'un avantage supplémentaire en faveur des savars / savirs / suvars en tant que tribu non turcque. Cependant, il faut se rappeler la nuance essentielle. Comme on le sait l'un des problèmes altaïques traditionnels et activement discutée est la question de la correspondance entre le $z$ et $r$ du turcque commun dans la branche bulgare (tchouvache) des langues turques, ainsi que dans les langues mongole et tungus-manchou. Cependant, les Altaïstes admettent que la thèse de la mise en contact des ancêtres des Tchouvaches "en Mongolie avec la Sibérie est une hypothèse non étayée par des preuves historiques" (Vasiliev, Chelim 1987: 76).

Il existe une distinction entre les premier et deuxième pronoms du singulier dans les langues turcque et tchouvache. Par exemple, à la première personne du singulier dans les langues turques, il y a des men et dans la langue tchouvache le эпӗ est nominatif. N.N. Poppe a noté que les pronoms en langue tchouvache se distinguent et ne peuvent être comparés directement aux pronoms turcque commun.

Certains pronoms en langue tchouvache sont complètement isolés. Et cela prouve encore une fois que les langues turcquophones sont beaucoup plus proches les unes des autres que la langue tchouvache. Selon N.N. Poppe, la langue tchouvache s'est isolée bien avant la formation de la langue pra-turcque (Poppe 1927: 61, 64). 
V.V. Radlov dans son recherche «Phonetik der Nördlichen Türksprachen» a écrit en particulier sur la transition des voyelles $y, u, a$ en $a$ dans les turcquismes. La première période est la transition de $b l(y)$ vers $a$ (par exemple, xblpăм $\rightarrow$ qaryn). À ce étape les mots ont été empruntés sous une forme très déformée. Deuxième période est la transition de $\mathrm{y}(u) \rightarrow \mathrm{a}$. Tchouvaches ont emprunté tellement de mots turcques au cours de cette période qu'ils ont commencé progressivement à prononcer correctement les sons turcques. Cependant, ils ont une forme déformée. Dans la troisième période, le Tchouvache $a$ commence à correspondre au $a$ Turcque. Ainsi, la prononciation des sons par les Tchouvash et les Turcs devient assez courante. Par conséquent, les Tchouvaches commencent à prononcer les mots turcques relativement vrais: кажӑк, кашкӑр, капкӑн, пазар (Radloff 1882: 90). «J'ai brièvement décrivé les impressions que j'avais eues lors de l'étude de l'attitude de la langue tchouvache vis-à-vis des langues turques dans la phonétique», - écrivait l'académicien. "Et maintenant, je crois que mon opinion précédente est petit à petit confirmée." «Tchuvash, comme Yakut, était à l'origine non turcophone, il est devenu turc avec le temps», - résume V.V. Radlov. Il a confirmé ces observations encore une fois dans un manuscrit inédit consacré à l'étude de la grammaire de la langue tchouvache, qu'il avait préparée pour publication, mais n'avait pas eu le temps de la publier. Pour la raison mystérieuse, son recherche n'est pas encore été publiés.

«Au cours des premiers siècles de contact avec le monde turcque, les Tchouvaches n'ont pas encore maitrisé l'habitude de parler à d'autres», a noté Vasily Vasilyevich. À ce list il include les mots que xblpăm «der Leib»=

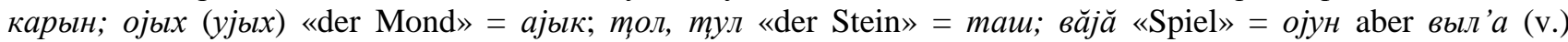
«spielen»= өiна (v.) (SPbF ARAN. F. 177. Op. 1. D. 33: 1-2, 25-26). Dans la «Classification des dialectes turcquophones» proposée par V.V. Radlov ne mentionne ni les langues tchouvaches ni les langues yakoutes, car les locuteurs de ces langues, à son avis, ne sont pas des Turcques mais des tribus turquisés. Sur cette propriété a déjà été portée l'attention (Kononov 1982: 211). N.N. Poppe a appelé V.V. Radlov est le représentant le plus important de la théorie de l'origine non turque du Tchouvache. V.V. Radlov considérait que la langue tchouvache dans son état actuel était le résultat du transfert du dialecte turcque sur la base non turcque (Poppe 1924: 293). Bien que V.V. Radlov n'a pas précisé le cadre chronologique des périodes mentionnées, il est clair que ces phénomènes proviennent de la formation du Kaganat turcque occidental, mais pas avant le milieu du 7ème siècle. Comme on le sait les Savirs vivaient dans le Caucase à cette époque.

Il faut accepter la position que les familles linguistiques nord-ouest (Abkhaz-Adyghé), nord-est (Nakh-Daghestan) et sud (Kartvel) sont indigènes en Caucase. Certaines langues indo-européennes et turques actuellement utilisées au Caucase ont atteint la région avec les tribus qui ont émigré plus tard. Par exemple, les ancêtres des Kumyks, les premiers habitants du Daghestan caspien, ont turcquifié tout le millénaire des VIe aux XVIe siècles.

On peut faire les observations intéressantes en comparant les langues karaite, bulgare et savir. Ces trois tribus avaient une communauté spatio-temporelle sur la côte de la mer Noire et ont relevé au conglomérat de l'État de Khazar. Par conséquent Azgar Mukhamadiev a estimé que «dans la langue karaïte, certains mots ont perdu ou changé la signification en bulgare (tatar)» (Mukhamadiev 2011: 85). Il en donne une longue liste (je cite des mots tchouvaches): аргъамакъ «coursier» (cavaliers en campagne assis sur la réserve куш am, et avant la bataille, ils l'ont remplacé par аргъамакъ) - tchouvache урхамах; ахиацы "Caissier" (apparemment, le mot avait la même prononciation dans la langue montante du Khazar) - tchouvache укс̧ас̧ӑ; балкъурт «Abeille»- tchouvache пыл хурт «miel» + «vermisseau»; кыркын «servante»- tchouvache хӑрхӑм; йэргэ «système»- tchouvache йӗрке; йэр-сув «patrimoine»- tchouvache çӗр-шыь «terre-eau»; ияардак «toit»- tchouvache чартак; иэлмэкии «гончар»- tchouvache чйллекс̧ее «potier» etc.

Les chercheurs s'intéressent également au son cliquetis de Mishars de Nijni Novgorod. En même temps ils associent ce phénomène à l'influence de Khazars en particulier des tribus de l'Alliance Saviro-Khazar. On peut en conclure que les ancêtres Mishars sort de la région du Caucase du Nord. Maintenant les Mishars vivent principalement dans la région de Kaibitsky au Tatarstan (appelée Molkeevskys), dans le district de Batyrevsky de la République de Tchouvache et dans la région de Nijni Novgorod.

On marque le remplacement du son $u$ dans la langue Mishar par le son $u$ dans la langue Kazan-Tatar. Par exemple, dans la lettre du tsar Joseph, on rencontre l'ethnonyme $u$-p-мис au lieu de la dernière forme de черемисы. En langue tchouvache, au lieu de $u$ et $u$, nous avons $c, c$ ou $u$. Cette liste est très longue et l'alternance des sons est cohérente (russe беглеи (évadé), mishar каикын, tatar качкын, tchouvache. каскӑн; russe горький (атеr), mishar äце, татар. ӓче, tchouvache йy̌çё; russe кричать (crier), mishar кыцкыр, tatar кычкыр, tchouvache кӑшкӑр; russe лещ (brème), mishar цабак, tatar чабак, tchouvache суупах; russe щука (brochet), mishar иуртан, татар. чуртан, tchouvache çăрттан).

En même temps il convient de noter que la formation de la langue des Tatars de Kazan a commencé avec l'apparition des Bulgares dans la Volga moyenne (Salmin 2017). Le fait que la langue bulgare appartient au groupe du son $k$ et que la langue tchouvache au groupe du son $x$ est également confirmé par le fait que les mots russoslaves et arabo-persans empruntés par les Bulgares étaient établis au son de $k$. Par exemple, соха $\rightarrow$ сука, хазина 
$\rightarrow$ казна (trésorerie) et le Suvaro-Chuvash - суха, хысна. Parmi d'autres faits est la transition Suvarsky дж à $\check{\text { et }}$ $p$ à 3. Ainsi, dans un épitaphe de la période bulgare on trouve les parallèles жиат жур et йите йоз (700 an.). Comme la plupart de chercheurs A.P. Kovalevsky a déclaré qu'après la majeure partie de la population du royaume bulgare ait commencé à parler le tatar comme résultat du renforcement de l'élément Kypchak cette langue devait être considérée comme la langue du groupe Kypchak-Tatar (Kovalevsky 1956: 7). Aux XIIIe et XIVe siècles la langue kypchake a pénétré dans la Bulgarie Volgienne et a «avalé» les Bulgares qui a donné naissance à la langue des Tatars Volgiens. La Bulgarie pendant la période d'Horde d'Or a reçu le statut de principauté vassale et se trouvait dans la sphère politique de Saray. Lorsque la langue kipchake était langue officielle la population bulgare a commencé à utiliser cette langue avec l'arabe dans les sphères intellectuelles. Et la question du nombre de Kipchaks vivant en Bulgarie à cette époque n'avait aucune importance. Par conséquent l'affirmation que la langue tatare s'est formée sur le substrat bulgaro-turc (Bereczki 1983: 207-236) est un fait évident. Cyro Hattori, cherchant la raison pour laquelle les Chuvash n'ont finalement pas adopté la langue turcqueo-tatar estime que les Suvars constituaient déjà une nation différente des Bulgares (Hattori 1980: 94).

L'abkhaze peut également témoigner de l'énorme influence de langue étrangère. Cela fait référence au séjour de deux cents ans des Turcs en Abkhazie, «à l'issue duquel presque tous les Abkhazes apprenaient turc qui est devenue la langue de communication avec les autres nations» (Gublia 2013: 4).

Le locuteur natifs de la langue kypchake sont entrés en Europe de l'Est au début du onzième siècle et les Tatars (Mongols-Tatars) sont apparus dans la région de Volga-Kama après l'invasion mongole. Maintenant, ces langues sont préservées par les Tatars et les Bachkirs de Kazan. En peu de temps les Kipchaks ont cohabité avec les tribus de langue tchouvache (Bulgares et Suvars) tandis que d'autres groupes tchouvaches ont vécu dans la région forestière où Volga se déplacent d'Est en Sud. Les Kypchaks ont utilisé turcque de la variante Khvazreze comme langue littéraire qui a rapidement été soumise à la forte influence de la langue locale. Les chercheurs distinguent un nombre de mots empruntés en bulgare (tchouvache) dans les langues des kipchaks de la Volga. Par exemple: tchouvache йе̌кер $\rightarrow$ ikiz («jumeaux»), tchouvache mănăp («balai») $\rightarrow$ sipir «balayer» (Agyagasi 2002: 26). Les langues hybrides Khvarezm-Turcque et Kipchake sont présentes sur les épitaphes des Bulgares de la Volga. Ces inscriptions sont parfois appelées inscriptions de premier type. Le second type contient des éléments caractéristiques des langues bulgare et kypchak-khvarezme. Le plus intéressant est la pierre tombale où l'épitaphe de la sépulcre est écrite d'un côté dans le premier style et de l'autre côté dans le second style.

La présence des Kypchaks dans les steppes de la région Oural-Volga était insignifiante à l'époque pré-mongole. Pour cette raison il n'est pas nécessaire de parler de leur influence sur le cours de la formation de la carte ethnique. Les contacts des nomades avec la population locale dans la seconde moitié du XIVe siècle indiquent la présence pendant la période de la Horde d'Or de sépultures nomades séparées dans des cimetières musulmans urbains et ruraux.

On devrait être d'accord avec B.A. Serebrennikov qui a déclaré que les Tchuvashs modernes sont l'ethnie qui maîtrisent la langue turcque étrangère ayant participé au processus de formation du peuple Tchuvash (Serebrennikov 1957: 41). À partir de ce moment commence le repliement du fonds lexicaux unique moderne des langues bulgaro-tatar et suvaro-tchouvache. Des mots comme jaš «vert», «jeune» deviennent courants. Cela inclut également le turcque commun jитүаq (јумБак) - «rond», «sphérique»; «écheveau»; jumšaq (jылмшак, јумшак, јумжак) - «doux, tendre». Al-Kashgarh йумга̄ «quelque chose en pente, rond» et un йумша̄к «quelque chose de mou» (Al-Kashgarh 2005: 774). Dans le premier cas le mot est entré dans Tchouvache avec un son retombé à la fin (çăмxa - "enchevêtrement", "balle"), et dans le second cas, le son final a été préservé (ŭăмшак - "douloureux"). On peut constater la charge sémantique a également changé. L'élision du $k$ final ont lieu dans le mot turcque qui est devenu tchouvache булак «point d'eau», «clé», «source»: la rivière Пӑла «Bula», le village de Пӑла cassia, bien qu'ils prononcent en même temps Пӑлак kassi (en prononciation пӑлаккасси) «Bulakovo». Comparez les noms des rivières à Kazan et dans le district de Batyrevsky: Булак - Була. La même chose s'est produite dans la langue tchouvache avec le mot turc kairak/kairaz̧: convertir $\mathrm{x} \rightarrow \mathrm{k}$ et la perte de la consonne finale en: hăyra «aiguisé». De même, cela s'est produit avec le mot tchouveshe mănpa «terre», «poussière de la terre»: à Kazan, la langue tatare est le- тупрак/топрак.

La ville de Simbirsk, située à l'époque bulgare sur la rive gauche de la Volga, s'appelait Çĕмnĕp et plus tard sur la rive droite Чёмпёр, c'est-à-dire que nous avons un exemple de transition du son $c$ à $\psi$. Même situation avec le mot tchouvache çухӑр "crier", "appeler" dans les dialectes ottomans et de Crimée de la langue turcque чақыр, чакыр, шакыр. Et aussi Tchuvash çy̆ç, turcque чач - "cheveux sur la tête d'une personne"; tchuvash çamau,

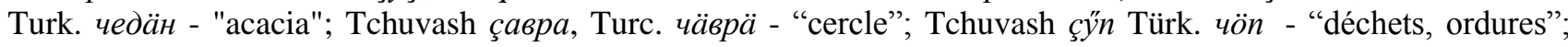
Tchuvash çе̌ре̌к, Turk. чйрy̋ - "pourri." Bien sûr, la première option est un mot purement tchouvache et la seconde est déjà transformée sous l'influence du discours turcque. En général les transformations phonétiques $c \rightarrow c \rightarrow u$

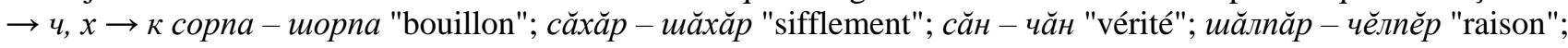
иийе - чие "cherry"), ainsi que les substitutions au niveau lexical çытар-минтер "oreiller") sont évidentes. 
Certain nombre de mots du tatar ayant passé dans la langue tchouvache sans modifications (moŭ/myŭ - «odeur», «avis», «sentir»; бum/num - «visage») (Fire 2015: 325, 383). Par conséquent, en ce qui concerne les Tchouvaches on peut affirmer que "ils ont endommagé leur langue tatare" (Tatichchev 1964: 67).

Les correspondances chuvash-balkares dans le vocabulaire ont également une place importante. Par exemple, Balkar. сокъур чеги, tchuvash суккацр пыршӑ (littéralement "véridique", "juge"); Balkar. Sokur Chegi, tchuvash sukkăr pyrsa (lit. "cecum"); Balkar. бюрче, tchuvash năpça «puce».

Il convient de mentionner le groupe non baptisé Tchouvache de Bakalinski (village de Yultimirovka et le village d'Akhmanovo) qui s'est transformé en langue tatare et a fusionné avec les Nagaibak locaux. Les Tchuvash est apparu dans cette région au plus tard que XVIIIİme siècle.

Comparé aux langues turcophones Tchuvash a changé beaucoup du type original. Cependant, certaines formes de cette langue sont de nature plus ancienne que les formes des dialectes turcophones. En comparant la langue tchouvache avec les dialectes turcques, N.I. Ashmarin a identifié plusieurs caractéristiques. Premièrement la loi sur l'harmonie des voyelles propre aux langues ouralo-altaïques n'est pas toujours strictement observée en tchouvache ou a un usage particulier (p. ex. en dialecte kurmysch). Deuxièmement, dans certains cas la racine d'un mot subit des changements qui ne sont pas du tout caractéristiques des dialectes turcophones. Troisièmement, les voyelles étroites des langues turcophones se transforment très souvent en larges parmi les tchouvaches, qui ne se trouvent dans aucune des langues turcophones. Quatrièmement, les consonnes de la langue tchouvache sont souvent doublées (à la fois en racines et en affixes) ce qui n'est pas retrouvé dans les langues turques (Ashmarin 1898: XIX XX). Bien sûr les différences incluent rotazim-zetatsizm (çăвap - aвblз «bouche»), năp - буз («glace»), хёp - қ̧blз «fille»), ainsi que la présence dans le vocabulaire tchouvach d'une couche significative de mots ceux absents dans les langues turcophones ( сӑмca - «nez»; çатмa - «poêle à frire»; тулӑ - «blé»).

Parlant du premier dirigeant de Маgуак Arpade, Konstantin Bagryanorodny l'appelait l'archonte de Turquie (Bagryanorodny 1991: 163). Également dans d'autres sources byzantines. Cependant, les Hongrois ont appelé leur terre Madjarorsag ce qui signifie littéralement "Pays Magyar", plutôt Uglish ou Ugria. Une source de 1253 indique que la langue du paskatir (bashkir) et du hongrois est la même (Rubruk 1997: 120). Bien que Rubruk ne précise pas ce qu'il veut dire (ugrisme ou turkisme bashkir),il est probable qu'il s'agisse d'éléments ougriens en langue bashkir. En 1236 le moine Julian a appelé les Bashkirs sur les Hongrois de l'Est du fleuve Etil car leur langue était le hongrois, ils le comprenaient et lui (Anninsky 1940: 73, 81). Mais la vérité est que la thèse de la parenté BachkirHongrois n'est pas confirmée par la linguistique (Zimonyi 2014: 150). Les auteurs arabes mentionnant les Magyars sous la forme de modjgar, il est fort probable qu'il s'agit de Mishars, bien que les Arabes aient à l'esprit les Bachkirs.

On sait que la langue hongroise conserve les premiers monuments de la langue turcque antique occidentale. Andras Rona-Tash a prouvé l'origine turcque occidentale de près de 400 mots hongrois. Par exemple le mot hongrois tenger "mer" dans toutes les langues turcques se présente sous la forme de tengiz, c'est-à-dire $z$ avec à la fin. Seulement le hongrois a conservé la forme ancienne. Il contient également 115 mots turcs occidentaux absents dans la langue tchouvache (Rona-Tash 2011: 136). La langue tchouvache contient moins de mots turcs occidentaux que le hongrois. Il se trouve qu'avant le Xème siècle les ancêtres des Tchouvaches ont beaucoup moins contacté les Turcs que les ancêtres des Hongrois. À cette époque les savirs étaient moins turcquisés que les Hongrois. En d'autres termes de plus nous nous plongeons dans l'histoire de moins il y a de similitudes linguistiques entre les Chuvash et les Turcque. La Tchouvache était turcquisée en Volga en empruntant aux langues kypchakes par l'intermédiaire des Tatars de Kazan et des Bachkirs. Selon les mots d'un linguiste quand il n'ya pas de sphères impénétrables de deux langues sans rapport il est pratiquement difficile de distinguer l'une de l'autre (Shcherbak 1994: 169).

Au début du XIIIème siècle les troupes mongoles-tatares envahissent l'Europe de l'Est avec des forces importantes. Kirakos Gandzaketsi (environ 1200-1271) a enregistré une liste de mots mongols entendus en Arménie. Par exemple: dieu- тангри, femme- эме, cheval- мори, chien- нохай, eau- усун, soleil- наран, etc. (Kirakos 1976: 173). Gustav Ramstedt, après avoir étudié à fond le vocabulaire et la phonétique a conclu que le développement de la langue tchouvache se déroulait sans contact direct avec la langue mongole (Ramstedt 1922: 34). En effet les mongols existants en langue tchouvache occupent une place insignifiante. Par exemple, la ville de Bulgar a été pendant quelque temps la seule dans les possessions de la maison de Jochi, où les pièces de monnaie des khans mongols ont été frappées. Cependant, des inscriptions sur les pièces de monnaie il est clair que la population au début du XIVe siècle a conservé son ancien langage dont les traces sont distinctes en tchouvache.

Dans le même temps, la population bulgare commence à être fortement influencée par le kypchak qui devient peu à peu la langue d'État ainsi qu'en Asie centrale. Andrash Rona-Tash a publié tous les emprunts en Mongol qu'ils ont remarqués en tchouvache. Il y a 29 mots dans le dictionnaire (selon NN Poppe, 33 mots). Par exemple: tchuvash.

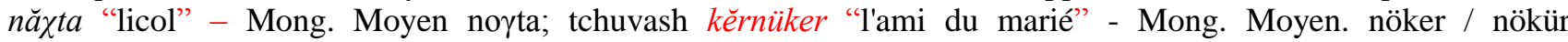
"combatant", "soldat"; tchuvash ұurčăka "faucon" - Mong. Moyen qarčiza. Sur ces 29 mots 17 sont trouvés en 
langue marie. Cela indique que la majorité des éléments chuvashs et tatars de la langue mari ont été empruntés après la période mongole, c'est-à-dire après XIIIe siècle. Leur intermédiaire principal était la langue tatare et la source de certains mots était tchouvache. Les mongolismes sont divisés en trois types: élevage de chevaux, chasse à l'aide d'oiseaux de proie, relations sociales (Róna-Tas 1973: 125-141). Dans son article N.N. Poppe conclut que les mots tchouvaches analysés par Andrash Rona-Tash n'ont pas été empruntés directement au mongol moyen, mais sont passés par les langues turcques voisines, principalement des Tatars de Kazan (Poppe 1977: 111-1114).

Cela devrait également inclure le mongol dalai "universel", "grand". Le mot est préservé comme archaïsme dans les chansons folkloriques tchouvaches. Par exemple: «С̧ут тецнче талайран юлнӑ». N.I. Ashmarin le définit comme un mot inconnu (Ashmarin 1937: 165). V.G. Egorov a traduit la phrase entière comme suit: «La terre (l'univers) a été préservée (a survécu) de l'océan» (Egorov 1964: 228-229). Ainsi, le mot mongol dalai apparaît chez les Tchouvaches sous la forme de талай au sens de "mer", "ocean". En hongrois talaj signifie "terre". Talaj est enregistré dans le texte de la légende turcque du XIIIe siècle à propos d'Oguz-kagan. Le mot tchuvash puyan «riche» est équivalent au mot mongol bayan. Tous ces faits montrent que le tchouvache est la langue la plus proche des langues mongoles parmi les langues turcqophones ce qui à certains égards révèle une nature archaïque. N.N. Poppe croyait que la langue tchouvache "peut avec quelques réserves être considérée comme un lien intermédiaire entre le turcque et le mongol" (Pope 1925: 31).

On peut voir que de nombreux chercheurs ont écrit sur la turcquisation des ancêtres tchouvaches. Donc, V.V. Radlov a affirmé que la langue tchouvache comme Yakut est devenu turcque, avec le temps (SPbF ARAN. F. 177, Op. 1. D. 33: 2). Cependant, il n'y a pas de recherche cohérente et systématique sur ce sujet. P. ex. on écrit: comme les autres populations locales finno-finlandaises ils étaient turcquisés, mais nous ne le savons pas (Novoseltsev 1990: 83). Bien sûr, la turcquisation a touché les Savirs dans le Caucase du Nord, mais ce processus a eu une place importante parmi les Tchuvash à l'époque de la Horde d'or, du Khaganat de Kazan et de la République du Tatarstan. À la lumière des relations linguistiques chuvash-turco-mongol, le point de vue valable de N.N. Poppe. À son avis la langue tchouvache a beaucoup en commun avec les adverbes turcques ainsi que de nombreuses similitudes avec la langue mongole. La caractéristique la plus significatif par laquelle la langue tchouvache diffère de la langue turcque est la présence, comme en mongol de* $\mathrm{p}$ de protolangue, ainsi que la correspondance de $l$ tchèques-mongole avec le $u$ turcque. Il est impossible de considérer la langue tchouvache comme turcque et comme mongole. La conclusion principale de N.N. Poppe: la langue tchouvache séparée des langues altaïques à l'époque pré-turcque (Pope 1925a: 425-426). Ou plutôt le путы qui parle tchouvache s'est séparé des langues ouralo-altaïennes à l'époque pré-turcique.

Très probablement la version des tribus «parlant le tchuvache» en Sibérie (personnes / groupes parlant le tchouvache) doit être citée, indiquant ainsi le caractère conventionnel du terme. En fait la turcquisation des ancêtres de la Tchouvache a commencé dans le Caucase et s'est déjà renforcée sur la Volga. Par conséquent il faut parler de l'influence de la langue du «peuple de langue tchouvache» (c'est-à-dire porteurs de signes $r, l$ et autres venus de Sibérie) aux ancêtres de la Tchouvache à travers les Bulgares-Tatars basés à Kipchkiz. Les chercheurs les appellent des "contes de Tchuvash" parce que les donneurs de langue eux-mêmes se sont dissous en destinataires. Si les Bulgares et les Savirs pouvaient conserver (sous condition) leur langue d'origine nous verrions alors que les Tatars modernes de Kazan et les Tchuvash (en tant que successeurs) d'origine n'ont à l'origine aucun rapport avec les Turcques y compris les Kipchaks. Cette hypothèse est également étayée par des informations tirées de l'histoire, de la géographie, de la génogéographie, de l'anthropologie, de l'archéologie, de la religion et d'autres sciences. Il s'avère que la langue turcque moderne de la langue tchouvache est un superstrat et en aucun cas un substrat, c'està-dire que ce processus a eu lieu (et continue de se produire) non pas en Sibérie, mais dans la région de la Volga. La langue turcque ancienne n'est fondamentalement conservée que dans le discours des tchouvaches modernes, ainsi les chercheurs pour la reconstruction des langues turcques à partir du XIIIe siècle le plus souvent recours aux données de la langue tchouvache. D'où la terminologie utilisée par les linguiste «groupes de langue chuvash». De plus cette vision de la turcquisation du Tchouvache n'est pas nouvelle. Écoutons le linguiste:

Après avoir mélangé les ancêtres du Tchouvache avec les Turcs, la langue tchouvache a maîtrisé la structure grammaticale turque et le vocabulaire de base turc et a commencé à se développer conformément aux lois internes des langues turcophones. Quant au vocabulaire et aux formes grammaticales pré-turques, ils ont peu à peu disparu et il ne reste plus que des traces misérables, uniques éléments lexicaux et grammaticaux. Jusqu'alors la linguistique est en route de la vérité (Egorov 1953: 77).

\section{Conclusion}

"La langue Tchuvashe comme Yakut n'était pas à l'origine turcophone", - comme V.V. Radlov a dit, - "il est devenu turcque avec le temps". L'académicien a confirmé ces observations encore une fois dans un manuscrit inédit consacré à l'étude de la grammaire de la langue tchouvache qu'il a préparé pour publication, mais n'a pas eu le 
temps de le publier. N.N. Poppe appelé V.V. Radlov comme le représentant le plus important de la théorie de l'origine non turcque du Tchouvache.

Il s'avère que la langue turcque moderne est un superstrat pour la langue tchouvache, et en aucun cas un substrat, c'est-à-dire que ce processus a eu lieu (et continue de se produire) non pas en Sibérie, mais dans la région de la Volga.

Après la conquête des Turco-Mongols des peuples de la Volga moyenne le processus de croisement progressif et de fusion des ancêtres de la Tchouvache avec les Turcque nomades migrés a commencé en un seul peuple avec une prédominance des Turcque et leur langue. Cependant, la kypchakisation en générale n'a pas affecté l'ethnogonie des Tchouvaches aux niveaux génétique et anthropologique.

\section{Références}

Agyagasi Klara. 2002. Some middle Bulgarian loan words in the Volga Kipchak languages B: Orientalia Academiae Scientiarum Hungaricae. Vol. 55 (1-3), p. 25-28.

Aidyn Erkhan. 2011. Zametki po povodu nazvanii tyurkskikh plemen, vstrechayushchikhsya v yeniseiskikh pamyatnikakh In: Epigrafika Vostoka. M.: IV RAN, str. 3-13.

Al-Kāshgari Makhmud. 2005. Divān Lugāt at-Turk. Per., predisl. i komment. - Z.-A.M. Auezova. Almaty: DaikPress.

Anninskii S.A. 1940. Izvestiya vengherskikh missionerov XIII i XIV vv. o tatarakh i Vostochnoi Yevrope In: Istoricheskii arkhiv. III. M.; L.: Izd-vo AN SSSR, str. 71-112.

Ashmarin N.I. 1898. Materialy dlya issledovaniya chuvashskogo yazyka. Ch. 1; Ch. 2. Kazan“: Tipo-lit. Imp. un-ta. Id. 1937. Slovar' chuvashskogo yazyka. Vyp. XIII. Cheboksary: Chuvashgosizdat.

Bagryanorodnyi Konstantin. 1991. Ob upravlenii imperiyei. Vvedeniye - G.G. Litavrin. [Grech.] tekst, per., komment. M.: Nauka.

Bartol'd V.V. 1968. Sochineniya. T. V. M.: Nauka.

Bereczki Gábor. 1983. A Volga-Káma vidék areális kapcsolatai In: Areális nyelvészeti tanulmányok. Budapest: Tankonyvkiado, p. 207-236.

Golden Peter B. 2006. Turks and Iranians: An Historical Sketch в: Turkic-Iranian Contact Areas: Historical and Linguistic Aspects. Wiesbaden: Harrassowitz, p. 17-38.

Id. 2011. Studies on the Peoples and Cultures of the Eurasian Steppes. Ed. by Cătălin Hriban. Bucureşti: Editura Academiei Române.

Gublia R.K. 2013. Ocherki po abkhazskoi etimologhii. Sukhum: AGU.

Dybo A.V. 2007. Lingvisticheskiye kontakty rannikh tyurkov: Leksicheskii fond: pratyurkskii period. M.: Vostoch. lit.

Id. 2015. Etimologhicheskii slovar' tyurkskikh yazykov. T. 9 (dopolnitel 'nyi): Etimologhicheskii slovar' bazisnoi leksiki tyurkskikh yazykov. M.: Nauka.

Yegorov V.G. 1953. K voprosu o proiskhozhdenii chuvash i ikh yazyka In: Zapiski. Vyp. VII. Cheboksary: NIIYaLI, str. 64-91.

Id. 1964. Etimologhicheskii slovar ' chuvashskogo yazyka. Cheboksary: Chuvash. kn. izd-vo.

Khattori S. 1980. O formirovanii tatarskogo i chuvashskogo yazykov In: Voprosy yazykoznaniya. N 3, str. 86-94.

Kirakos Gandzaketsi. 1976. Istoriya Armenii. M.: Nauka.

Kononov A.N. 1982. Istoriya izucheniya tyurkskikh yazykov v Rossii: Dooktyabr'skii period. L.: Nauka.

Kovalevskii A.P. 1956. Kniga Akhmeda Ibn-Fadlana o yego puteshestvii na Volgu v 921-922 gg. St., per. i komment. Khar'kov: Khar'kov. un-t.

Kyzlasov Leonid. 1997. Pervyi tyurkskii kaganat i yego znacheniye dlya istorii Vostochnoi Yevropy In: Tatarskaya arkheologhiya. Kazan', N 1, str. 23-30.

Mukhamadiyev Azgar. 2011. Novyi vzglyad na istoriyu gunnov, khazar, Velikoi Bulgarii i Zolotoi Ordy. Kazan': Tatar. kn. izd-vo.

Nadelyayev V.M., Nasilov D.M., Tenishev E.R., Shcherbak A.M., red. 1969. Drevnetyurkskii slovar'. L.: Nauka.

Novosel'tsev A.P. 1990. Khazarskoye gosudarstvo i yego rol'v istorii Vostochnoi Yevropy i Kavkaza. M.: Nauka.

Poppe N.N. 1924. Chuvashskii yazyk i yego otnosheniye k mongol'skomu i turetskim yazykam In: Izvestiya Rossiiskoi akademii nauk. VI seriya. T. XVIII, str. 289-314.

Id. 1925. O rodstvennykh otnosheniyakh chuvashskogo i tyurko-tatarskikh yazykov. Cheboksary: Chuvashoblizdat.

Id. 1925a. Chuvashskii yazyk i yego otnosheniye k mongol'skomu i turetskim yazykam In: Izvestiya Rossiiskoi akademii nauk. VI seriya. T. XIX, str. 405-426.

Id. 1927. Ukazatel'nyye i voprositel'nyye mestoimeniya v chuvashskom yazyke In: Vestnik nauchnogo obshchestva tatarovedeniya. N 7. Kazan', str. 61-64.

Id. 1977. Chuvash-Mongolian Linguistic Contacts In: Journal of the American Oriental Society 97.2, p. 111-114.

Radloff W. 1882. Phonetik der Nördlichen Türksprachen. Leipzig: T.O. Weigel. 
Ramstedt G.J. 1922. Zur Frage nach der Stellung des Tschuwassischen B: Journal de la Société Finno-Ougrienne. XXXVIII. Helsinki, p. 1-34.

Róna-Tas András. 1970. Some Problems of Ancient Turkic In: Acta Orientalia Havniae. 32, p. 209-228.

Id. 1973-1974. Középmongol eredetü jövevényszavok a csuvasban II In: Néprajz és Nyelvtuomány. 17-18, p. 125141.

Id. 2007. Nutshell Chuvash URL: httpwww2.lingfil.uu.seafroturkiskasparakIP2007NUTSHELLCHUVASH.pdf.pdf

Id. 2011. Ot Urala do Karpatskogo basseina: (Novyye rezul'taty issledovanii po rannei istorii vengrov) In: Altaistika i tyurkologhiya. N 4 (4), str. 131-139.

Rtveladze E.V. 2015. $\triangle$ APENH 'ATPOחON: Marshrut vizantiiskogo posol'stva Zimarkha po Srednei Azii i Kavkazu In: Problemy istorii, filologhii, kul'tury. 1, str. 354-364.

Rubruk Ghil'om de. 1997. Puteshestviye v Vostochnyye strany In: Karpini Dzhovanni del Plano. Istoriya mongalov. Rubruk Ghil'om de. Puteshestviye v Vostochnyye strany. Polo Marko. Kniga. Per. - I.M. Minayev. M.: Mysl'‘, str. 88-189.

Salmin A.K. 2017. History of the Bulgars: from Armenia to Kazan In: Bulgarian historical Review. N 1-2, p. 3-17.

Serebrennikov B.A. 1957. Proiskhozhdeniye chuvash po dannym yazyka In: O proiskhozhdenii chuvashskogo naroda. Cheboksary: Chuvashgosizdat, str. 28-47.

Shcherbak A.M. 1994. Vvedeniye v sravnitel 'noye izucheniye tyurkskikh yazykov. SPb.: Nauka.

$\mathrm{SPbF}$ ARAN (The Archive of the Russian Academy of Sciences St. Petersburg Branch). 1916-1918. F. 177. Op. 1. D. 33 - W.W. Radlow. [Die Grammatik der Sprache Chuvash. Ne opublikovano].

Takazov F.M., sost. 2015. Digorsko-russkii slovar'. Vladikavkaz: B.i.

Tatishchev V.N. 1964. Istoriya Rossiiskaya. T. 4. M.; L.: Nauka.

Thomsen Vilhelm. 1924-5. Alttürkische Inschriften aus der Mongolei In: Zeitschrift der Deutschen Morgenländischen Gesellschaft. Bd. 78. Lpz., p. 121-175.

Vasil'yev D.D., Khelimskii Ye.S. 1987. XXIX sessiya Postoyannoi mezhdunarodnoi altaisticheskoi konferentsii In: Vestnik Akademii nauk SSSR. N 5, str. 72-78.

Vitsen Nikolaas. 2010. Severnaya $i$ Vostochnaya Tartariya, vklyuchayushchaya oblasti, raspolozhennyye v severnoi $i$ vostochnoi chastyakh Yevropy i Azii. T. II. Amsterdam: Pegasus, S. 622-1225.

Zhivkov Boris. 2015. Khazaria in the Ninth and Tenth Centures. Leiden; Boston: Brill.

Zimonyi István. 2007. History of the Turkic speaking peoples in Europe before the Ottomans URL: http://www2.lingfil.uu.se/afto/turkiskasprak/IP2007/ZimonyiIP.pdf

Id. 2014. Medieval Nomads in Eastern Europe: Collected Studies. Bucureşt: Brăila.

\section{RÉSUMÉ}

La langue contient également une histoire. Pour l'analyse il convient de comparer les langues et les langues des tribus et des peuples voisins. La langue dans le temps peut être divisée en plusieurs langues descendantes. Cela est dû à la violation de l'unité de la société dans laquelle elle opère. Lorsque les différences commencent à entraver la compréhension mutuelle entre les membres de la société, des langages différents mais apparentés apparaissent. Le changement est un attribut obligatoire de la langue parce qu'un système gelé ne pourrait pas assurer la communication de la société dans un monde en mutation. Les linguistes (AV Dybo et autres) ont établi une vitesse d'érosion de 5 mots de la liste de Swadesh depuis 1000 ans dans un certain nombre de groupes linguistiques. Avec une telle rapidité de plus de 20000 ans, la liste de Swadesh devrait être complètement effacée. Il s'avère qu'aucun langage statique n'existe depuis 20000 ans.

La langue turque pour la langue moderne tchouvache est le superstrat, et en aucun cas substrat, c'est-à-dire que ce processus a eu lieu (et continue de se dérouler) non en Sibérie, mais dans la région de la Volga. 